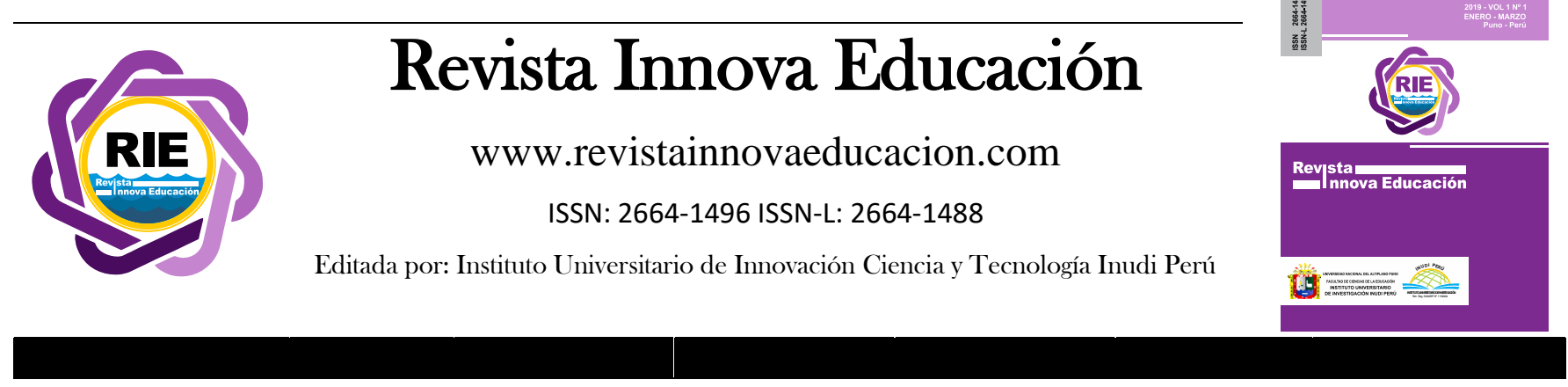

\title{
Percepción del clima de aula universitaria por los estudiantes de la Escuela Profesional de Educación Secundaria
}

\author{
Perception of the classroom climate by the students of the Secondary School Professional School \\ Alfredo Castro $^{1}$ (D); Sara Farfán Cruz ${ }^{1}$; Karen Ortega ${ }^{1}$; Pierina Velezvia ${ }^{1}$ \\ DOI: https://doi.org/10.35622/j.rie.2019.01.008
}

${ }^{1}$ Universidad Nacional del Altiplano, Perú

Recibido el 21/01/2019/ Aceptado el 27/01/2019

\begin{tabular}{|c|c|}
\hline $\begin{array}{l}\text { ARTÍCULO ORIGINAL } \\
\text { PALABRAS CLAVE } \\
\text { Clima de aula, } \\
\text { gestión de clase, } \\
\text { satisfacción, } \\
\text { evaluación, cohesión. }\end{array}$ & $\begin{array}{l}\text { La investigación tiene como propósito describir la percepción que muestran los } \\
\text { estudiantes respecto al clima de aula universitaria. La población y muestra de estudio } \\
\text { está compuesta por los estudiantes de la Escuela Profesional de Educación Secundaria. } \\
\text { El tipo de investigación es descriptivo, el diseño es descriptivo diagnóstico, la técnica } \\
\text { empleada es la encuesta, el cuestionario se denomina clima de aula universitaria, siendo } \\
\text { de tipo licker, el cual consiste en un conjunto de ítems aseverativas expresadas en } \\
\text { categorías que fueron respondidas por los encuestados. Se sistematiza la información } \\
\text { recogida en tablas y gráficos estadísticos, utilizándose la medida porcentual en su } \\
\text { lectura e interpretación, considerando las escalas de acuerdo (A) y completamente de } \\
\text { acuerdo (CA). Los resultados nos muestran que respecto a las dimensiones: cohesión, } \\
\text { satisfacción, personalización, orientación a la tarea, innovación, evaluación y gestión } \\
\text { de clase, se evidencia una medida porcentual menor del } 62 \% \text { que corresponde a la } \\
\text { dimensión orientación a la tarea y una medida porcentual mayor del 79\% que } \\
\text { corresponde a la dimensión personalización. En suma, las dimensiones en estudio } \\
\text { muestran un clima favorable de aula universitario. }\end{array}$ \\
\hline $\begin{array}{l}\text { KEYWORDS } \\
\text { Classroom climate, } \\
\text { class management, } \\
\text { satisfaction, } \\
\text { evaluation, cohesion. }\end{array}$ & $\begin{array}{l}\text { The purpose of the research is to describe the perception that students show regarding } \\
\text { the climate of the university classroom, the population and study sample is composed } \\
\text { of students of the Secondary School Professional School, the type of research is } \\
\text { descriptive, the design is descriptive, diagnostic, the technique used is the survey, the } \\
\text { questionnaire is called university classroom climate, being of the licker type, which } \\
\text { consists of a set of assertive items expressed in categories that were answered by the } \\
\text { respondents. The information collected in statistical tables and graphs is systematized, } \\
\text { using the percentage measure in its reading and interpretation, considering the scales } \\
\text { of agreement (A) and completely in agreement (CA), the results show us that regarding } \\
\text { the dimensions: cohesion, satisfaction, personalization, task orientation, innovation, } \\
\text { evaluation and class management, a percentage measurement of less than } 62 \% \text { is shown } \\
\text { that corresponds to the dimension oriented to the task and a percentage measure greater } \\
\text { than } 79 \% \text { corresponding to the personalization dimension. In sum, the dimensions } \\
\text { under study show a favourable classroom climate. }\end{array}$ \\
\hline
\end{tabular}




\section{INTRODUCCIÓN}

El clima de aula universitario es una expresión compleja nada fácil de definir debido a que encierra múltiples dimensiones de estudio, en ella confluyen una parte material tales como el mobiliario, la infraestructura por citar, y por otra, inmaterial que incluye a las personas, sus características, la interacción entre estudiantes, entre docentes y estudiantes. El aula en sí constituye un espacio donde interactúan estudiantes y docentes-estudiantes, dependiendo de esa interacción algunos pueden afirmar que se evidencia un buen clima de aula o que no lo es para otros; pues bien, se trata de percepciones distintas y por esa razón es que resulta un tanto complejo. Biggs (2015) al referirse al clima de la enseñanza y el aprendizaje sostiene "cada profesor, como cada institución en su conjunto, crea un clima de aprendizaje mediante las interacciones formales e informales con los alumnos. Este clima tiene que ver con la forma que ellos y nosotros tenemos de sentir las cosas y esto, naturalmente, tiene efectos positivos o negativos sobre su aprendizaje". Pues lo que perseguimos al experimentar experiencias de aprendizaje en el aula es el logro de aprendizajes que deben contribuir a nuestra formación profesional, sea a través de exposiciones, diálogos o hechos inesperados que puedan suscitarse en su desarrollo.

La investigación centra su estudio en un primer grupo que considera las siguientes dimensiones: Cohesión, satisfacción y personalización, las cuales se expresan a través de la relación de amistad que existe entre los estudiantes, en el trabajo que se realiza en el aula resulte interesante y divertido, las oportunidades generadas por el docente que provoquen interacciones activas y promuevan su aprendizaje; un segundo grupo está referido a la orientación a la tarea, el cual está centrado en el desarrollo personal del estudiante y debe visualizarse en el compromiso de permanecer en la asignatura sacando provecho en la solución de problemas; y por último, un tercer grupo conformado por: innovación, evaluación y gestión de clase, los cuales se relacionan con el cambio y mantenimiento del sistema, se deben expresar mediante el desarrollo del pensamiento creativo de los estudiantes, se explicita las normas de control de los estudiantes en el aula, el docente informa a los alumnos de los procedimientos necesarios para efectivizar la enseñanza.

El estudio explicita también los materiales y métodos, en ella se considera el tipo y diseño de investigación utilizada, la población y muestra, las técnicas e instrumentos de recolección de datos, el plan de tratamiento estadístico de los datos. También presenta los resultados y su discusión respecto a la variable de estudio; las conclusiones y las referencias bibliográficas.

\section{MÉTODOS Y MATERIALES}

El tipo de investigación es descriptivo, en ella se observan los fenómenos tal y como se dan en su contexto natural, para después analizarlos; además, "la investigación no experimental o expost-facto es cualquier investigación en la que resulta imposible manipular variables o asignar aleatoriamente a los sujetos o a las condiciones" (Kerlinger, F. \& Lee, 2002). De hecho, no hay condiciones o estímulos a los cuales se expongan los sujetos del estudio. El diseño de investigación es el descriptivo diagnóstico, este diseño va a ponderar las características esenciales del clima de aula por los estudiantes. 
La población y muestra de estudio está compuesta por los estudiantes de las especialidades de Ciencias Sociales y Matemática, Física, Computación e Informática matriculados en el año académico 2017 siendo en número 57 estudiantes. En este tipo de muestra, "el investigador procede a seleccionar la muestra en forma intencional, eligiendo aquellos elementos que considera convenientes y cree que son los más representativos" (Carrasco, 2006).

En relación con la variable de estudio clima de aula se utilizó la técnica de la encuesta, siendo su instrumento el cuestionario denominado clima de aula universitaria, el cual facilitó el acopio de información respecto a la variable en estudio. El instrumento que se aplicó corresponde a (Villa, L.), quien realizó una investigación a estudiantes de universidades españolas considerando las siete dimensiones que se asumió, para lo cual se hizo algunas adecuaciones contextuales necesarias. Se sistematizó la información mediante la presentación de tablas y gráficos estadísticos, además, se empleó la medida porcentual con la finalidad de cuantificar los resultados alcanzados.

\section{RESULTADOS}

\section{Presentación de resultados}

A continuación, presentamos los resultados obtenidos al aplicar el cuestionario de clima de aula universitaria a los estudiantes de las especialidades de Matemática, Física, Computación e Informática y Ciencias Sociales de la Escuela Profesional de Educación Secundaria, el instrumento consta de 7 dimensiones y cada uno de 7 ítems, haciendo un total de 49 ítems respectivamente.

El cuestionario propuesto considera frases que describen el clima o ambiente psicosocial de la clase de esta asignatura, en ella no hay respuestas "verdaderas" ni "falsas", solamente opiniones, cada declaración contiene cuatro posibles respuestas:

$\mathrm{CD}=\mathrm{Si}$ estás completamente en desacuerdo que la frase describe el ambiente real de esta clase.

$\mathrm{D}=\mathrm{Si}$ estás en desacuerdo que la frase describe el ambiente real de esta clase.

$\mathrm{A}=\mathrm{Si}$ estás de acuerdo que la frase describe el ambiente real de esta clase.

$\mathrm{CA}=\mathrm{Si}$ estás completamente de acuerdo que la frase describe el ambiente real de esa clase.

Luego de la utilización de la escala según los datos obtenidos, se sistematizó y procedió a la lectura e interpretación de los datos en tablas y gráficos estadísticos, utilizándose para ello la medida porcentual en relación con la variable de estudio clima de aula.

Resultados obtenidos de clima de aula universitaria en los estudiantes de la Escuela Profesional de Educación Secundaria

Tabla 1 Grado de cohesión entre estudiantes, docente-estudiantes de la Escuela Profesional de Educación Secundaria

\begin{tabular}{lcccccccc} 
Escala & $\begin{array}{l}\text { Completamente } \\
\text { desacuerdo }(\mathbf{C D})\end{array}$ & en & $\begin{array}{c}\text { En desacuerdo } \\
(\mathbf{D})\end{array}$ & $\begin{array}{l}\text { De } \\
\text { (A) }\end{array}$ & $\begin{array}{l}\text { Acuerdo } \\
\text { Completamente } \\
\text { acuerdo (CA) }\end{array}$ & Tetal \\
\hline Puntuación & 40 & & 83 & 164 & 112 & 399 \\
$\%$ & 10 & & 21 & 41 & 28 & 100
\end{tabular}

Fuente: Cuestionario de clima de aula universitaria

La tabla 1 presenta información respecto a la amistad y confianza entre estudiantes, entre docentesestudiantes. El 10\% de estudiantes manifiesta estar completamente en desacuerdo (CD), el $21 \%$ 
de ellos afirma que están en desacuerdo (D), el $41 \%$ indican que están de acuerdo (A) y el 28\% sostiene que están completamente de acuerdo (CA).

Por lo cual, considerando las dos puntuaciones últimas, podemos afirmar que el $69 \%$ de los estudiantes manifiestan una relación favorable entre ellos y entre docentes-estudiantes, el cual repercute en un clima de aula favorable.

Tabla 2 Grado de satisfacción en el trabajo de clase por los estudiantes de la Escuela Profesional de Educación Secundaria

\begin{tabular}{lccccc} 
Escala & $\begin{array}{c}\text { Completamente } \\
\text { en desacuerdo } \\
\text { (CD) }\end{array}$ & $\begin{array}{c}\text { En desacuerdo } \\
\text { (D) }\end{array}$ & $\begin{array}{c}\text { De acuerdo } \\
\text { (A) }\end{array}$ & $\begin{array}{c}\text { Completament } \\
\text { e de acuerdo } \\
\text { (CA) }\end{array}$ & Total \\
\hline Puntuación & 25 & 86 & 174 & 114 & 399 \\
$\%$ & 6 & 22 & 44 & 28 & 100
\end{tabular}

Fuente: Cuestionario de Clima de Aula Universitaria

En la tabla se presenta información respecto al grado de satisfacción que muestran los estudiantes respecto al trabajo que realizan en la clase. El 6\% de estudiantes manifiestan estar completamente en desacuerdo (CD), el 22\% de ellos afirman que están en desacuerdo (D), el $44 \%$ indican que están de acuerdo (A) y el 28\% sostienen que están completamente de acuerdo (CA).

Por lo tanto, considerando las dos puntuaciones últimas, podemos afirmar que el $72 \%$ de los estudiantes manifiestan un grado de satisfacción positiva en el trabajo de clase, el cual favorece un buen clima de aula.

Tabla 3 Grado de interacción entre docente-estudiante en el aula, en la Escuela Profesional de Educación Secundaria

\begin{tabular}{lccccr} 
Escala & CD & D & A & CA & Total \\
\hline Puntuación & 28 & 55 & 191 & 125 & 399 \\
$\%$ & 7 & 14 & 48 & 31 & 100
\end{tabular}

Fuente: Cuestionario de Clima de Aula Universitaria

En la tabla 3 da a conocer la interacción que se da en el aula entre docentes y estudiantes. El 7\% de estudiantes manifiesta estar completamente en desacuerdo (CD), el 14\% de ellos afirma que están en desacuerdo (D), el 48\% indican que están de acuerdo (A) y el 31\% sostiene que están completamente de acuerdo (CA).

Por lo cual, considerando las dos puntuaciones últimas, podemos afirmar que el $79 \%$ de los estudiantes manifiestan que se evidencia una interacción armoniosa entre docentes y estudiantes en el trabajo que se realiza en clase, el cual en definitiva favorece a un buen entendimiento y clima de aula.

Tabla 4 Grado de cumplimiento de actividades por los estudiantes de la Escuela Profesional de Educación Secundaria

\begin{tabular}{lccccc} 
Escala & CD & D & A & CA & Total \\
\hline Puntuación & 54 & 99 & 163 & 83 & 399 \\
$\%$ & 13 & 25 & 41 & 21 & 100
\end{tabular}

Fuente: Cuestionario de Clima de Aula Universitaria 
La tabla 4 presenta información respecto al cumplimiento de las actividades por parte de los estudiantes en clase. El 13\% de estudiantes manifiesta estar completamente en desacuerdo (CD), el 25\% de ellos afirma que están en desacuerdo (D), el $41 \%$ indican que están de acuerdo (A) y el $21 \%$ sostiene que están completamente de acuerdo (CA).

Por lo cual, considerando las dos puntuaciones últimas, podemos afirmar que el $62 \%$ de los estudiantes asumen el cumplimiento de las actividades en clase, el cual favorece un buen clima de aula.

Tabla 5 Grado de trabajo creativo en clase por los estudiantes de la Escuela Profesional de Educación Secundaria

\begin{tabular}{lccccc} 
Escala & CD & D & A & AC & Total \\
\hline Puntuación & 23 & 77 & 201 & 98 & 399 \\
$\%$ & 6 & 19 & 50 & 25 & 100
\end{tabular}

Fuente: Cuestionario de Clima de Aula Universitaria

La tabla 5 presenta información respecto a las diversas actividades que presenta el docente en el aula para desarrollar el pensamiento creativo de los estudiantes. El 6\% de estudiantes afirman estar completamente en desacuerdo (CD), el 19\% de ellos indican que están en desacuerdo (D), el 50\% evidencian que están de acuerdo (A) y el 25\% sostienen que están completamente de acuerdo (CA).

Por lo tanto, considerando las dos puntuaciones últimas, podemos afirmar que el $75 \%$ de los estudiantes manifiestan que las actividades desarrolladas en el aula favorecen a la creatividad de ellos, el cual se evidencia en un buen clima de aula.

Tabla 6 Grado de control en el aula a los estudiantes de la Escuela Profesional de Educación Secundaria, semestre 2017

\begin{tabular}{cccccc} 
Escala & CD & D & A & CA & Total \\
\hline Puntuación & 31 & 78 & 181 & 109 & 399 \\
$\%$ & 8 & 20 & 45 & 27 & 100
\end{tabular}

Fuente: Cuestionario de Clima de Aula Universitaria

La tabla 6 muestra información respecto al grado de control que ejerce el docente en la clase respecto a los estudiantes. El $8 \%$ de estudiantes manifiesta estar completamente en desacuerdo (CD), el 20\% de ellos afirma que están en desacuerdo (D), el $45 \%$ indican que están de acuerdo (A) y el $27 \%$ sostiene que están completamente de acuerdo (CA).

Por lo tanto, considerando las dos puntuaciones últimas, podemos afirmar que el $72 \%$ de los estudiantes manifiestan que los docentes muestran un control apropiado en relación con el comportamiento de los estudiantes en la clase, el cual ayuda a un buen clima de aula.

Tabla 7 Grado de organización en el aula por los docentes respecto a los estudiantes de la Escuela Profesional de Educación Secundaria

\begin{tabular}{lccccc} 
Escala & CD & D & A & CA & Total \\
\hline Puntuación & 25 & 86 & 174 & 114 & 399
\end{tabular}


Fuente: Cuestionario de Clima de Aula Universitaria

La tabla 7 da a conocer el grado de organización que establecen los docentes en relación con los estudiantes en el aula. El 8\% de estudiantes indica estar completamente en desacuerdo (CD), el $20 \%$ de ellos asevera que están en desacuerdo (D), el 47\% indican que están de acuerdo (A) y el $25 \%$ afirman que están completamente de acuerdo (CA).

Por lo tanto, asumiendo las dos puntuaciones últimas, podemos afirmar que el $72 \%$ de los estudiantes manifiestan que los docentes muestran un grado de organización apropiado en la clase, el cual favorece un buen clima.

\section{DISCUSIÓN}

El propósito de la investigación está orientado a describir la percepción de los estudiantes de la Escuela Profesional de Educación Secundaria respecto a las dimensiones de la variable clima de aula universitaria. La categoría relación comprende tres dimensiones: cohesión, satisfacción, y personalización; la primera dimensión está referida al grado de amistad y confianza entre los estudiantes, esto resulta importante puesto que permite la interacción entre los estudiantes en el aula; respecto al grado de confianza entre los estudiantes "se afirma que la conversación entre niños, en la que intentan explicar sus métodos, contribuye a su propia comprensión. Deberíamos distinguir claramente entre la conversación cuando es una charla inútil y la conversación que aporta significado al aprendizaje matemático" (Gorgorió, 2000); los resultados alcanzados respecto a la dimensión cohesión nos muestran que el 69\% de los estudiantes manifiestan una relación favorable entre ellos y entre docentes-estudiantes, de hecho que cuando en el aula se evidencia elementos comunicativos, éstos fortalecen la amistad, la confianza; por lo cual, podemos afirmar que un clima de aula favorable fortalece la confianza, el diálogo, el trabajo en equipo que muestran los estudiantes y tendrá efecto en su aprendizaje. Respecto a la dimensión satisfacción, ésta se concibe como un sentimiento de bienestar o placer que experimenta uno cuando alcanza un deseo o cubre una necesidad; al respecto, se sostiene que "en una tutoría o sesión de grupo en la que el tutor censure o se muestre sarcástico, los estudiantes permanecerán en silencio, prefiriendo no hacerse vulnerables"(Biggs, 2015), como podrá verse, esto no resulta favorable a los intereses de los estudiantes. Los resultados respecto a la dimensión satisfacción evidencian que el $72 \%$ de los estudiantes manifiestan un grado de satisfacción positiva en el trabajo de clase, el cual favorece un buen clima de aula. Respecto a la dimensión personalización, está referida a la interacción entre docentes y estudiantes, en ella "la interacción profesor-alumno, desarrollada por el profesor con la intención de contribuir al aprendizaje del alumno es una forma de llevar a la práctica la evaluación formativa"(Giménez, J. \& Santos, L. \& Da Ponte, 2004), del mismo modo que, "el aprendizaje entre iguales o entre colegas, (...), es una forma de organizar el proceso de enseñanza en el salón de clases que considera como aspecto esencial el trabajo de los alumnos en algún momento del desarrollo de la lección en pequeños grupos" (Ferreiro, R. \& Espino, 2009). Los resultados obtenidos en relación con la dimensión personalización nos muestran que el 79\% de los estudiantes manifiestan que se evidencia una interacción armoniosa entre docentes y estudiantes en el trabajo que se realiza en clase; como se verá en definitiva fortalece una buena relación y un buen clima de aula. 
La dimensión orientación a la tarea comprende la categoría desarrollo personal, al respecto se sostiene que "en una asignatura basada en problemas y, por lo demás, muy buena, de una determinada universidad, una tutora arruinó por completo el proceso. El objetivo es que los estudiantes planteen preguntas e investiguen las respuestas verosímiles a un problema dado"(Biggs, 2015), pues lo que se pretende es que los estudiantes desarrollen sus propias habilidades resolutivas y la función del docente debe ser orientador del proceso de aprendizaje. En esta dimensión los resultados nos muestran que el $62 \%$ de los estudiantes manifiestan el grado de cumplimiento de las actividades que desarrollan en clase, el cual favorece al estudiante que no solo espera ser formado, sino él mismo se forme como profesional, por lo que, genera un buen clima de aula.

La categoría cambio y mantenimiento en el sistema comprende tres dimensiones: innovación, evaluación, y gestión de clase. La primera dimensión está referida al conjunto de actividades que planifica el docente con miras a desarrollar el pensamiento creativo de los estudiantes; se asume que "el pensamiento creativo es la capacidad que permite generar ideas novedosas, interesantes y con originalidad para resolver problemas que plantea la vida cotidiana y académica" (Torres, 2007), en ese sentido, se asume que el pensamiento original es un proceso mental que nace de la imaginación, desconociéndose el modo en que difieren las estrategias mentales, el pensamiento convencional y el creativo, pero la cualidad de la creatividad puede ser valorada por el resultado final. En relación con la dimensión innovación los resultados expresan que el $75 \%$ de los estudiantes manifiestan que las actividades desarrolladas en el aula favorecen a la creatividad de ellos, el cual se evidencia en un buen clima de aula. En relación con la dimensión evaluación, consideramos de importancia debido a que en todo momento de nuestras vidas estamos evaluando si lo que pretendemos es mejorar; asumimos la evaluación como el proceso de recojo y análisis de información, la emisión de un juicio valorativo y toma de decisiones; "Las investigaciones demuestran que los educandos aprenden a emplear el tiempo asignado. Si terminan antes, hay que recordarles revisar cuidadosamente el trabajo o enriquecerlo, o pensar mejor la presentación que se hará del mismo. La ayuda mutua entre los miembros del equipo se acrecienta en función del tiempo disponible" (Ferreiro, R. \& Espino, 2009), asimismo, "en una evaluación al servicio del aprendizaje, la comunicación adquiere un papel fundamental. En especial, el cuestionamiento es ciertamente un proceso poderoso para que el profesor ayude al alumno a regular su aprendizaje cuando desarrolla su trabajo en el aula" (Gimenez, J. \& Santos, L. \& Da Ponte, 2004). Ahora bien, respecto a la dimensión evaluación, los resultados sostienen que el $72 \%$ de los estudiantes manifiestan que los docentes muestran un control apropiado en relación con el comportamiento de los estudiantes en la clase, el cual ayuda a un buen clima de aula, por lo que, si el propósito es la mejora constante, entonces el proceso evaluativo es fundamental. Con referencia a la dimensión gestión de clase, el docente organiza el trabajo en el aula y consensua las normas de convivencia, como se verá "es importante que el profesorado fomente los procesos comunicativos, orales o escritos, entre estudiantes para generar reflexiones sobre las resoluciones y sobre la gestión de las mismas" (Gimenez, J. \& Santos, L. \& Da Ponte, 2004). Los resultados en relación con la dimensión gestión de clase expresan que el $72 \%$ de los estudiantes manifiestan que los docentes muestran una organización apropiada en clase y asumen acuerdos de convivencia; por lo cual en definitiva favorece un buen clima de aula. 


\section{CONCLUSIONES}

Las dimensiones cohesión, satisfacción y personalización están unidos mediante la categoría relación y evidencian una puntuación medio-alto, expresado en un 73\%. Éstas se refieren al grado de amistad y confianza entre estudiantes, ellos notan que sus ideas son escuchadas y valoradas y, la interacción entre docentes y estudiantes es favorable a los intereses de aprender.

La dimensión orientación a la tarea se relaciona con la categoría desarrollo personal y muestra una puntuación medio-alto, expresado en un $62 \%$, ésta se refiere al grado de responsabilidad de permanencia en el aula y resolución de problemas que muestran los estudiantes.

Las dimensiones innovación, evaluación y gestión de clase se relacionan con la categoría cambio y mantenimiento en el sistema y evidencian una puntuación medio-alto, expresado en un $73 \%$, éstas se refieren al rol del docente en la planificación de actividades tendientes a desarrollar la creatividad de los estudiantes, el docente hace explícito el propósito de la asignatura que se desarrolla y, la organización y normatividad sobre el desarrollo de la asignatura.

\section{REFERENCIAS BIBLIOGRÁFICAS}

Abdón, I. (2003). Aprendizaje y desarrollo de las competencias. (C. editorial Magisterio, Ed.). Bogotá.

Alfaro, B. y Capella, J. y Córdova, M. (2009). Gestión del conocimiento. (I. G. S. R. SAC, Ed.). Lima - Perú.

Biggs, J. (2015). Calidad del aprendizaje universitario. (N. S. de Ediciones, Ed.). Madrid.

Carrasco, S. (2006). Metodología de la investigación. (E. S. Marcos, Ed.). Lima - Perú.

Corbalán, F. (2006). La matemática aplicada a la vida cotidiana. (S. Edit. Graó, de IRIF, Ed.). España.

Díaz, F. \& Hernández, G. (1999). Estrategias docentes para un aprendizaje significativo. (M.-H. E. S. de CV, Ed.). México.

Durán, G. (n.d.). (PDF) Principios de Aprendizaje Efectivo: Contexto de la Clase de Matemática II. Retrieved from https://www.researchgate.net/publication/257360656_Principios_de_ Aprendizaje_Efectivo_Contexto_de_la_Clase_de_Matematica_II

Évano, C. (2006). La gestión mental. (E. GRAÓ, Ed.). España.

Ferreiro, R. \& Espino, M. (2009). El ABC del aprendizaje cooperativo. (E. T. S. de CV, Ed.). México.

Gimenez, J. \& Santos, L. \& Da Ponte, J. (2004). La actividad matemática en el aula. (S. Edit. GRAÓ de IRIF, Ed.). España.

Gorgorió, N. \& O. (2000). Matemática y Educación. (S. Edit. GRAÓ de IRIF, Ed.). Barcelona.

Hernández, R. \& Fernandez, C. \& Baptista, P. (2010). Metodología de la investigación. (S. de C. McGraw-Hill/Interamericana Editores, Ed.). México.

Kerlinger, F. \& Lee, H. (2002). Investigación del comportamiento. (E. M. H. I. editores S. de CV, Ed.). México. 
L., V. (n.d.). diseno_instrumento.pdf. Retrieved January 12, 2017, from http://espacio.uned.es/fez/eserv/bibliuned:20337/diseno_instrumento.pdf

Lizarzaburu, A. \& Zapata, G. (2001). Pluriculturalidad y aprendizaje de la matemática en américa latina. (M. SL, Ed.). Madrid.

Lorenzo, M. (1999). El liderazgo educativo en los centros docentes. (E. L. M. SA, Ed.). Madrid.

MINEDU. (n.d.). Compromiso de maestro: formación en la práctica. Lima - Perú.

MINEDU. (2009a). Diseño curricular nacional de educación básica regular. Lima - Perú.

MINEDU. (2009b). Participación y clima institucional. Lima - Perú.

Morín, E. (2007). Los siete saberes necesarios a la educación del futuro. (E. magisterial servicios Gráficos, Ed.). Lima - Perú.

Ríos, D. \& Bozzo, N. \& Marchant, J. \& Fernández, P. (2010). Factores que inciden en el clima de aula universitario. Retrieved March 17, 2017, from http://www.redalyc.org/articulo.oa ?id $=27018888004$

Romero, C. (2007). La escuela media en la sociedad del conocimiento. (E. N. Educativas, Ed.). Lima - Perú.

Sáez, C. (2017). El buen clima en el aula: una propuesta para su instauración. Universidad Internacional de la Rioja. Retrieved from https://reunir.unir.net/bitstream/handle/ 123456789/4958/SAEZ GUILLEN\%2C CRISTINA.pdf?sequence=1\&isAllowed=y

Sánchez, H. (2007). Cómo desarrollar el pensamiento creativo. (E. V. Universitaria, Ed.). Lima Perú.

Thales, S. (1991). Estándares curriculares y de evaluación para la educación matemática. Sevilla.

Torres, A. (2007). Educación matemática y desarrollo del pensamiento matemático. (E. Rubiños, Ed.). Lima - Perú. 